\title{
Multimode fibre probe calibration
}

\author{
Petr Ják1 ${ }^{1,}$, Martin Šiler ${ }^{1}$, Jan Ježek ${ }^{1}$, Johanna Trägårdh $^{1}$, Tomáš Čižmár ${ }^{1,2}$, Pavel Zemánek ${ }^{1}$ \\ ${ }^{1}$ Institute of Scientific Instruments of the CAS, v.v.i., Královopolská 147, 61264 Brno, Czech republic \\ ${ }^{2}$ Leibniz Institute of Photonic Technology, 07745 Jena, Germany
}

\begin{abstract}
Multimode fibres (MMF) used in endoscopy have advantage of small diameter and flexibility, thus causing less damage to living animals. However, the imaging requires wavefront shaping techniques to obtain a sharp image despite the mode dispersion in the waveguide. We suggest version of transmission matrix calibration which uses internal modes of the waveguide and, thus, lessens requirements on the endoscopy apparatus removing the external reference path
\end{abstract}

\section{Endoscopy imaging}

The prevalence of neurodegenerative diseases connected with an increasing age of the population requires preclinical studies of animal models to develop a knowledge base of neuronal processes and blood flow deep in the brain. Especially experiments in vivo are of immense importance for the future of the research of Alzheimer's, Parkinson's and other diseases in order to translate the results to human medicine [1]. Measurements in brain tissue require access to the deep layers, but the penetration of microscopy techniques do not go deeper than approximately $1.5 \mathrm{~mm}$. Endoscopy probes come in different sizes and imaging quality. From several millimetres in diameter (GRIN lenses) [2], over fibre bundles [3] to multimode optical fibres (MMF) [4]. Especially the latter are of increasing popularity for their narrow footprint and flexibility. Obtaining an image using MMF probe is not straightforward, though. Due to different group velocities of the fibre modes, the phases are mixed and resulting image is an apparently chaotic distribution of speckles.

To characterize the fiber and achieve successful imaging, it is necessary to map the system response described by a transmission matrix (TM) $[5,6]$. The calibration process requires a highly homogeneous reference beam in order to extract modes' phases using phase-shifting interferometry. Although a high quality matrix (as well as resulting endoscopic images) is obtained, the experimental setup is somewhat complicated by the necessity of introduction of a reference beam in the calibration part of the apparatus and the overall phase stabilization of the optical paths not to introduce artefacts in the measurement.

A suggested alternative calibration technique uses internal modes of the endoscopic probe as a reference for TM calibration. However, the intensity distribution of the reference beam in the sample plane is a speckle pattern which results in blind spots in the measured TM due to lack of interference signal in the reference mode intensity minima. One of the previous ideas was to run the calibration procedure second time with another mode set as a reference field. Since the speckle patterns are different, it is possible to use the second measurement to cover the holes in TM [7]. Our approach is a little bit different. Running several procedures, each with different single internal reference mode, we obtain $\mathrm{N}$ "holey" TMs. Every output mode is defined as a superposition of all the input modes with phase and amplitude measured with reference $R_{1}$ in the first measurement. The same output mode measured with reference $R_{n}$ is reconstructed from input modes with a constant phase shift $\Delta \varphi=\varphi_{R 1}-\varphi_{R n}$; the value of $\Delta \varphi$ is revealed by dot product of the input mode vectors. Combined TM is then created by adding all the particular $\mathrm{TMs}$ to $\mathrm{TM}_{1}$ where the added columns are shifted by $\Delta \varphi$ and negated.

Although the calibration procedure is time consuming due to repetition of the calibration task with different references, it results in a high quality homogeneous image.

\section{Experimental results}

The experimental setup requires a device for wavefront shaping (a spatial light modulator SLM in our case), transformation optics to project the Fourier plane of SLM to the MMF probe input facet and an imaging path to observe the output modes of the probe. We have used

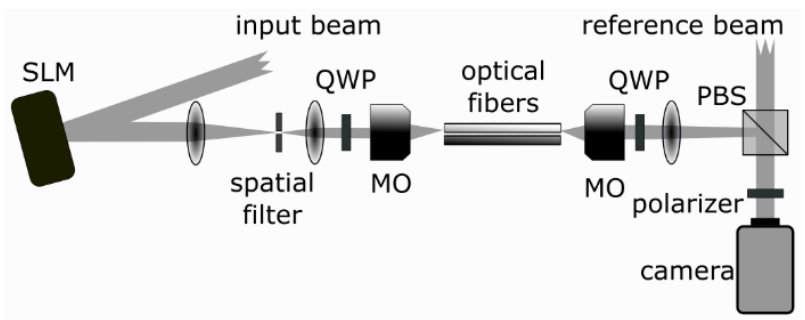

Fig. 1 : Experimental apparatus with spatial light modulator (SLM), quarter waveplates (QWP) and microscope objectives (MO). 
circularly polarized light (one polarization state only), as it is less sensitive to crosstalk with the other polarization state. An infrared laser $(\lambda=1070 \mathrm{~nm})$ and an optical fibre with $\mathrm{NA}=0.22, \mathrm{~d}=50 \mu \mathrm{m}$ was used. The external reference beam is introduced to setup for comparison and it is not used during calibration with internal references.

To estimate the performance of the internal calibration technique, we have measured the intensity of all the output modes addressed one by one. The values were put together to form an image of output fibre facet for quick visual check and their uniformity was expressed using the interference contrast formula. We have characterized the results from calibration with randomly chosen references. Obviously, the more internal

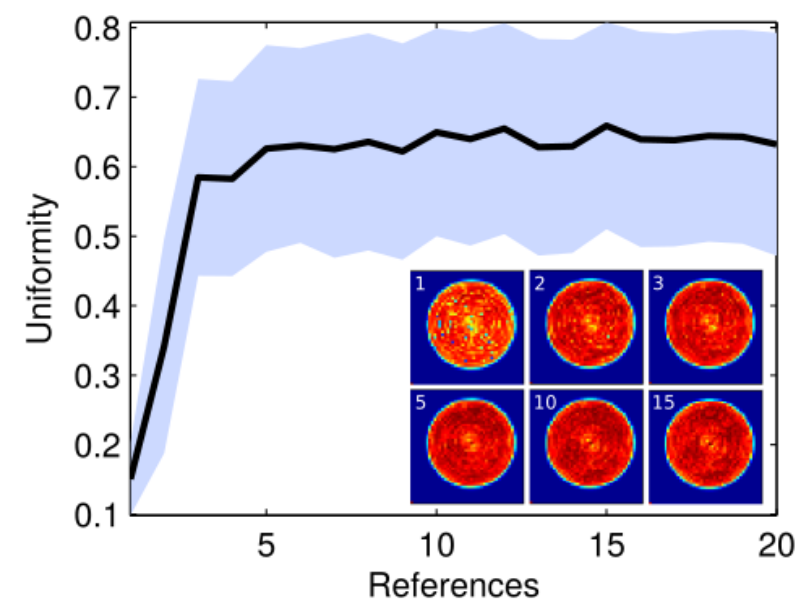

Fig. 2 :Uniformity measurement for 1-20 randomly chosen internal references. Inset shows intensity of all output modes across the fiber facet to visually compare homogenity.

references we used, the less blind spots or fluctuations in the output mode intensity we observed. Surprisingly, it was saturated quickly, as more than five references rarely had any effect on the uniformity.

We have used the calibrated endoscope to record images of 1951 USAF resolution test prepared by electron beam lithography in a chromium layer. The target was placed approximately $10 \mu \mathrm{m}$ behind the output facet, the output modes were addressed one by one and the detector further down the optical path collected light passing through. This way, we have simulated the endoscopy

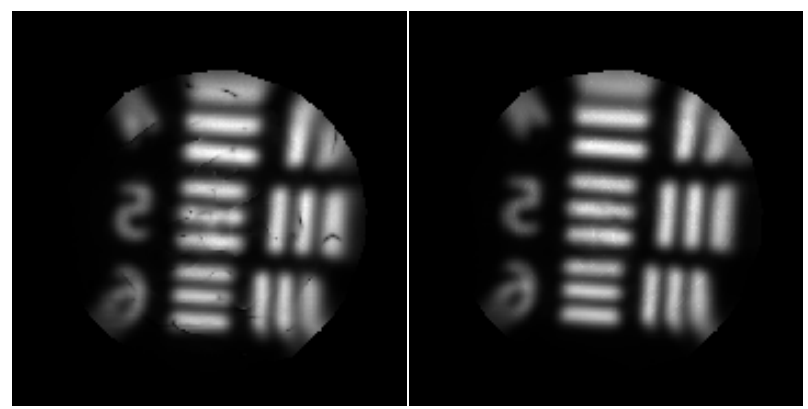

Fig. 3 :Endoscopic imaging of USAF target. System calibrated with 1 (left) and 5 (right) internal references. imaging, where the fluorescence signal is collected by the probe itself and passed to the detector in the setup.

\section{Conclusion}

We have demonstrated calibration and imaging with multimode fibre probe. The calibration itself used internal modes of the fibre and removed the necessity to stabilize the external reference path. The price for it is longer time of the procedure, as the technique requires several runs of measurements of the transmission matrix. The resulting calibration is highly uniform, especially when calibrated with 5 or more references, as we have proved by imaging the USAF target in an endoscopic configuration.

\section{References}

1. P. McGonigle, Biochem. Pharmacology 87, 140 (2014)

2. J. Jung, A. D. Mehta, E. Aksay, R. Stepnoski, M. J. Schnitzer, J. Neurophysiology 92, 3121 (2004)

3. S. P. Mekhail, N. Abudukeyoumu, J. Ward, G. Arbuthnott, S. N. Chormaic, Biomedical Optics Express 9, 1843 (2018)

4. S. Turtaev, I. T. Leite, T. Altwegg-Boussac, J. M. P. Pakan, N. L. Rochefort, T. Cizmar, Light: Sience \& Applications 7 (2018).

5. S. Popoff, G. Lerosey, R. Carminati, M. Fink, A. Boccara, S. Gigan, Phys. Rev. Lett. 104 (2010).

6. T. Cizmar, K. Dholakia, Opt. Express 19, 18871 (2011).

7. S. Bianchi, V. P. Rajamanickam, L. Ferrara, E. Di Fabrizio, C. Liberale, R. Di Leonardo, Opt. Lett. 38, 4935 (2013) 